\title{
ERROR BOUNDS FOR THE NUMERICAL INTEGRATION OF FUNCTIONS WITH LIMITED SMOOTHNESS
}

\author{
KAI DIETHELM ${ }^{\dagger \ddagger}$
}

\begin{abstract}
Recently, Trefethen (SIAM Review 50 (2008), 67-87) and Xiang and Bornemann (SIAM J. Numer. Anal. 50 (2012), 2581-2587) investigated error bounds for $n$-point Gauss and Clenshaw-Curtis quadrature for the Legendre weight with integrands having limited smoothness properties. Putting their results into the context of classical quadrature theory, we find that the observed behaviour is by no means surprising and that it can essentially be proved for a very large class of quadrature formulas with respect to a broad set of weight functions.
\end{abstract}

Key words. Numerical integration; Gauss quadrature; Clenshaw-Curtis quadrature; error bound; bounded variation; weight function.

AMS subject classification. 41A55

The numerical approximation of the weighted integral

$$
I_{w}[f]:=\int_{-1}^{1} w(x) f(x) \mathrm{d} x
$$

with a nonnegative integrable weight function $w$ and $f$ possessing certain smoothness properties is a classical topic of research 1, 2 that recently attracted new attention. Specifically, Trefethen [11 looked at the $n$-point formulas of Gauss type $Q_{n}^{\mathrm{G}}$ and Clenshaw-Curtis type $Q_{n}^{\mathrm{CC}}$, respectively, for $w \equiv 1$ and showed assuming that $f^{(s-1)}$ is absolutely continuous for some $s \in\{1,2,3, \ldots\}$, $\operatorname{Var} f^{(s)}<\infty$ (where $\operatorname{Var} g$ denotes the total variation of $g$ over the interval $[-1,1]$ ) and that $f^{(s)}$ is sufficiently well behaved near the points \pm 1 , cf. [11, p. 75 and Theorems 4.5 and 5.1], that

$$
I_{w}[f]-Q_{n}^{\mathrm{CC}}[f]=O\left(n^{-s}\right) \quad \text { and } \quad I_{w}[f]-Q_{n}^{\mathrm{G}}[f]=O\left(n^{-s}\right) .
$$

Later, without requiring Trefethen's assumptions about the behaviour of the integrand near the boundary, Xiang and Bornemann [12] improved the bounds to

$$
I_{w}[f]-Q_{n}^{\mathrm{CC}}[f]=O\left(n^{-s-1}\right) \quad \text { and } \quad I_{w}[f]-Q_{n}^{\mathrm{G}}[f]=O\left(n^{-s-1}\right) ;
$$

however, their proof for the Gauss formula needs the additional restriction that $s \geq 2$.

We now want to place these results into the context of classical quadrature theory. In this connection, a quadrature formula is a linear functional $Q$ of the form $Q[f]:=$ $\sum_{j=1}^{n} a_{j} f\left(x_{j}\right)$ with nodes $-1 \leq x_{1}<x_{2}<\ldots<x_{n} \leq 1$ and weights $a_{j} \in \mathbb{R}$. Following the common terminology in numerical integration 2 , we say that such a quadrature formula is positive if $a_{j} \geq 0$ for all $j$, and a formula is interpolatory if $Q[p]=I_{w}[p]$ for all $p \in \mathcal{P}_{n-1}$, the set of all polynomials of degree not exceeding $n-1$.

To achieve our goal we define the function classes

$$
V_{0}:=\{f:[-1,1] \rightarrow \mathbb{R}: \operatorname{Var} f<\infty\}
$$

and

$V_{s}:=\left\{f:[-1,1] \rightarrow \mathbb{R}: f^{(s-1)}\right.$ is absolutely continuous and $\left.\operatorname{Var} f^{(s)}<\infty\right\}$ for $s \in \mathbb{N}$

\footnotetext{
${ }^{\dagger}$ GNS Gesellschaft für numerische Simulation mbH, Am Gaußberg 2, 38114 Braunschweig, Germany, diethelm@gns-mbh.com

${ }^{\ddagger}$ Institut Computational Mathematics, Technische Universität Braunschweig, Fallersleber-TorWall 23, 38100 Braunschweig, Germany, k.diethelm@tu-bs.de
} 
and recall an important result of Freud [5, Satz II] (see also [6, §III.4] for the case $s=0$ ) who proved, using an argument based on one-sided polynomial approximation in the $L^{1}$ norm, the following statement:

THEOREM 1. Let $Q_{n}$ be a positive interpolatory quadrature formula with $n$ nodes for the weight $w$, and assume $w(x) \leq M\left(1-x^{2}\right)^{-1 / 2}$ for some $M>0$. Then,

$$
\left|I_{w}[f]-Q_{n}[f]\right| \leq 5 M((s+2) \pi)^{s+1}(s !)^{-1} n^{-s-1} \operatorname{Var} f^{(s)}
$$

whenever $f \in V_{s}$ for some $s \in \mathbb{N}_{0}$.

Freud's result has some immediate consequences:

1. The sharper bounds (3) hold for all functions $f$ with an absolutely continuous derivative of order $s-1$ and $\operatorname{Var} f^{(s)}<\infty$ for all $s \in \mathbb{N}_{0}$ (again without additional assumptions near the boundary of the interval).

2. Bounds of the same order also hold for a very large class of weight functions and many other quadrature formulas including, e.g., the Gauss and Radau formulas for all admissible weight functions [1, 2], the formulas of Clenshaw-Curtis [3] and their close relatives due to Filippi [4] and Polya [10, at least for the standard weight function $w \equiv 1$, and the Gauss-Kronrod formulas 7, 8, for the ultraspherical weight functions $w_{\lambda}(x)=\left(1-x^{2}\right)^{\lambda-1 / 2}$ where $\lambda \in[0,1] \cup\{3\}$ (cf. also 9]).

Other traditional techniques from the theory of numerical integration provide additional insight. For example, positive interpolatory quadrature formulas are not the only numerical integration methods admitting an error estimate of the form (3) for functions $f \in V_{s}$ : At least for the Legendre weight function $w \equiv 1$, compound quadrature schemes exhibit the same behaviour. To see this, let $Q$ be an arbitrary quadrature formula given by $Q[f]=\sum_{j=1}^{\ell} a_{j} f\left(x_{j}\right)$. We then subdivide the basic interval $[-1,1]$ into $n$ subintervals of length $2 / n$, affinely transform $Q$ to each of these subintervals and add up the resulting formulas, thus obtaining the $n$-fold compound quadrature formula with respect to the elementary formula $Q$, denoted and defined by

$$
Q^{(n)}[f]:=\frac{1}{n} \sum_{\nu=1}^{n} \sum_{j=1}^{\ell} a_{j} f\left(-1+\frac{1}{n}\left(x_{j}+2 \nu-1\right)\right) .
$$

Using the Peano kernel representation of the associated error functional (cf. 1, Satz 17, Satz 93 and Satz 97] or [2, $\S \S 4.2$ and 4.4]), one can immediately conclude

THEOREM 2. Let $w \equiv 1$, let $Q^{(n)}$ be the $n$-fold compound quadrature formula for the elementary formula $Q$, and assume that $Q[p]=I_{w}[p]$ for all $p \in \mathcal{P}_{m}$ with some $m \in \mathbb{N}_{0}$. Then, for every $s \in\{0,1, \ldots, m\}$ there exists a constant $C$ (depending only on $Q$ and s) such that, for all $f \in V_{s}$,

$$
\left|I_{w}[f]-Q^{(n)}[f]\right| \leq C n^{-s-1} \operatorname{Var} f^{(s)} .
$$

Thus, Xiang's and Bornemann's improvements of Trefethen's estimates for the error of Gaussian or Clenshaw-Curtis quadrature for integrands having an sth derivative of bounded variation fit into a more general picture.

\section{REFERENCES}

[1] Brass, H. (1977): Quadraturverfahren. Vandenhoeck \& Ruprecht, Göttingen.

[2] Brass, H., and Petras, K. (2011): Quadrature Theory. Amer. Math. Soc., Providence, RI.

[3] Clenshaw, C. W., and Curtis, A. R. (1960): A method for numerical integration on an automatic computer, Numer. Math. 2, pp. 197-205. 
[4] Filippi, S. (1964): Angenäherte Tschebyscheff-Approximation einer Stammfunktion - eine Modifikation des Verfahrens von Clenshaw und Curtis, Numer. Math. 6, pp. 320-328.

[5] Freud, G. (1955): Über einseitige Approximation durch Polynome, I, Acta. Sci. Math. 16, pp. $12-28$.

[6] Freud, G. (1971): Orthogonal Polynomials. Pergamon Press, Oxford.

[7] Gautschi, W. (1988): Gauss-Kronrod quadrature - A survey. In Milovanović, G. V. (ed.), Numerical Methods and Approximation Theory III. University of Niš, Faculty of Electronic Engineering, Niš, pp. 39-66.

[8] Kronrod, A. S. (1964): Nodes and Weights for Quadrature Formulae. Sixteen Place Tables. Nauka, Moscow (in Russian); English translation (1965): Consultants Bureau, New York, NY.

[9] Petras, K. (1999): Positivity of Gauss-Kronrod formulae for a certain ultraspherical weight function. In Gautschi, W., Golub, G. H., and Opfer, G. (eds.): Applications and Computation of Orthogonal Polynomials. Birkhäuser, Basel, pp. 195-202.

[10] Polya, G. (1933): Über die Konvergenz von Quadraturverfahren, Math. Z. 37, pp. 264-286.

[11] Trefethen, L. N. (2008): Is Gauss quadrature better than Clenshaw-Curtis? SIAM Rev. 50, pp. $67-87$.

[12] Xiang, S., and Bornemann, F. (2012): On the convergence rates of Gauss and Clenshaw-Curtis quadrature for functions of limited regularity, SIAM J. Numer. Anal. 50, pp. 2581-2587. 